\title{
Association between the frequency of toothbrushing and lifestyle in people with type 2 diabetes mellitus: at the baseline date of the Kamogawa-DM cohort study
}

Yuto Saijo, ${ }^{1}$ Hiroshi Okada, ${ }^{1, *}$ Masahide Hamaguchi, ${ }^{2}$ Takuro Okamura, ${ }^{2}$ Yoshitaka Hashimoto, ${ }^{2}$ Saori Majima, ${ }^{2}$ Takafumi Sennmaru, ${ }^{2}$ Naoko Nakanishi, ${ }^{2}$ Emi Ushigome, ${ }^{2}$ Mai Asano, ${ }^{2}$ Masahiro Yamazaki, ${ }^{2}$ and Michiaki Fukui ${ }^{2}$

1Department of Diabetes and Endocrinology, Matsushita Memorial Hospital, 5-55 Sotojima-cho, Moriguchi 570-8540, Japan 2Department of Endocrinology and Metabolism, Kyoto Prefectural University of Medicine, Graduate School of Medical Science, 465 Kajii-cho, Kawaramachi-Hirokoji, Kamigyo-ku, Kyoto 602-8566, Japan

(Received 28 January, 2021; Accepted 15 March, 2021; Published online 28 May, 2021)

\begin{abstract}
It has been reported that oral health is associated with some co-morbid conditions, including cardiovascular disease, in people with type 2 diabetes mellitus. The present study investigated the association between the frequency of toothbrushing and lifestyle in people with type 2 diabetes mellitus. This cross-sectional study included 624 outpatients at the Kyoto Prefectural University of Medicine in Kyoto, Japan from January 2014 to January 2016. Lifestyle was evaluated using a self-administered questionnaire. The average age and hemoglobin A1c level were $67.6 \pm 10.9$ years and $7.2 \pm 1.1 \%$, respectively. The number of patients who brushed their teeth twice or more a day was 189 (50.3\%) in men and 198 $\mathbf{( 7 9 . 8 \% )}$ in women. Among men, the proportion of patients living alone was lower in those who brushed their teeth twice or more a day than those who brushed their teeth never/rarely or once a day. The logistic regression analysis, after adjusting for confounding factors, revealed that living alone (odds ratio $\mathbf{2 . 8 8}$; $95 \%$ confidence interval 1.53-5.66) was associated with the increased odds of the low frequency of toothbrushing (never/ rarely or once a day) in men, but not in women. In conclusion, the results of our study suggest that living alone was associated with the low frequency of toothbrushing in people with type 2 diabetes mellitus, particularly in men.
\end{abstract}

Key Words: frequency of toothbrushing, living alone, type 2 diabetes

\footnotetext{
Cardiovascular disease (CVD) is the primary cause of mortality and morbidity in people with type 2 diabetes mellitus, and several risk factors, such as hypertension, smoking, and dyslipidemia have been proven to prompt the progression of CVD. ${ }^{(1,2)}$ Several studies have reported the association between oral health, specifically periodontal disease, and CVD. ${ }^{(3)}$ Periodontal disease results from a chronic inflammation of the tissue surrounding the teeth. Oral infections due to periodontal disease may contribute to the inflammatory burden and increase the risk of CVD. ${ }^{(4)}$

As we already know, diabetes is in close relationship with periodontal disease. ${ }^{(5)}$ A previous study has shown that diabetes is positively correlated with the risk of periodontal disease after adjusting for several confounding factors. ${ }^{(5)}$ The study ${ }^{(5)}$ reported that the rate of periodontal disease in people with type 2 diabetes mellitus was $10 \%$ higher than those without. Demmer et al. ${ }^{(6)}$ have reported that periodontal status was associated with the levels of hemoglobin A1c in patients without diabetes.
}

Kobayashi et al. ${ }^{(7)}$ have suggested that frequent toothbrushing is linked to a lower prevalence and incidence of metabolic syndrome. Further, self-reported oral hygiene behavior, such as the frequency of toothbrushing, has been reported to be associated with $\mathrm{CVD}^{\left({ }^{(8)}\right.}$ It follows from these reports that encouraging patients to brush their teeth might help them achieve better glycemic control. A few studies ${ }^{(8)}$ have reported the relation between oral health and lifestyle habits, such as smoking, in general population. It is important to investigate what kind of patients we should pay more attention to and what we need to know about the patients' backgrounds, which in turn helps us provide a better quality of care. As far as we know, there is no study that reports the association between the frequency of toothbrushing and lifestyle in people with type 2 diabetes mellitus. Therefore, we are the first to look into this association, including lifestyle parameters like marital status and living alone, in those patients.

\section{Materials and Methods}

Ethics. This study was approved by the local research ethics committee of Kyoto Prefectural University of Medicine (approval number RBMR-E-466-1) and has been conducted in accordance with the ethical principles of the Declaration of Helsinki. All patients provided written informed consent prior to the enrolment in the study.

Patients and study design. We started the KAMOGAWADM cohort study in 2014 to investigate the natural history of patients with diabetes. This was a cross-sectional study conducted in 624 outpatients at the Kyoto Prefectural University of Medicine (Kyoto, Japan) from January 2014 to January 2016. All data for the present study were retrieved from the database. We excluded the following patients from our study: (i) those who used steroid treatment, (ii) those who attended a hospital for psychosis, (iii) patients with malignancy, (iv) patients with severe renal dysfunction (estimated glomerular filtration rate $<30$ $\mathrm{ml} / \mathrm{min} / 1.73 \mathrm{~m}^{2}$ ), (v) patients who had undergone a renal or liver transplantation, and (vi) patients with the missing data.

Questionnaire and measurements. We collected the information on preferences and lifestyle using the self-administered questionnaire, which was standardized and has been previously

*To whom correspondence should be addressed.

E-mail: conti@koto.kpu-m.ac.jp 
validated. Five items regarding lifestyle were included. For example, a positive answer to the question of "Are you living alone?" is defined as living alone. Patients were classified into non-smokers, past smokers, or current smokers; and into coffee, tea, or alcohol drinkers, who daily drank coffee, tea, or alcohol, respectively. The patients who did any sport once a week regularly were defined as regular exercisers. ${ }^{(9)}$ Fasting blood samples were collected in the morning to assess blood glucose, hemoglobin A1c, total cholesterol, triglycerides, uric acid, and creatinine. Total serum cholesterol and triglyceride levels were measured using the standard enzymatic methods. Diagnosis of diabetes was based on the report issued by the expert committee on the diagnosis and classification of diabetes. ${ }^{(10)}$ Calculation of BMI was based on participant self-report by the questionnaire.

Statistical analyses. To evaluate the association between the patients' characteristics and the frequency of toothbrushing, we categorized the patients according to the frequency of toothbrushing (never/rarely, once a day, or twice or more a day), referring to the criteria adopted in a previous study. ${ }^{\left({ }^{(}\right)}$We defined hypertension as taking medication for hypertension. We defined hyperlipidemia as total cholesterol level $\geq 220 \mathrm{mg} / \mathrm{dl}$ and/or triglycerides' level $\geq 150 \mathrm{mg} / \mathrm{dl}$, or taking medication for hyperlipidemia. Owing to the differences in the lifestyle between men and women, the patients were stratified by sex to evaluate the association between lifestyle and the frequency of toothbrushing. The associations among the variables were assessed by an unpaired Student's $t$ test, ANOVA or the chi-square test. We defined never/rarely or once a day as low frequency of toothbrushing according to previous study. ${ }^{(8)}$ Logistic regression analysis was conducted to examine the effects of preference and lifestyle on low frequency of toothbrushing (never/rarely or once a day). The following confounding factors were taken into consideration: smoking habit, alcohol consumption, and living alone (Model 1). In addition to all variables in Model 1, Model 2 included age, body mass index, hemoglobin A1c level, hypertension, and hyperlipidemia as the cofounding factors.

All continuous variables are presented as mean (SD) or absolute number. $P$ value $<0.05$ was considered as statistically significant. The size, direction, and statistical significance of relationships were estimated by the odds ratio with $95 \%$ confidence interval (CI). Statistical analyses were performed using JMP 10 (SAS Institute, Cary, NC).

\section{Results}

The characteristics of 624 patients enrolled in this study are shown in Table 1. The average age and hemoglobin A1c level were $67.6 \pm 10.9$ years and $7.2 \pm 1.1 \%$, respectively. The number of patients who brushed their teeth twice or more a day was 189 $(50.3 \%)$ in men and $198(79.8 \%)$ in women. The women brushed their teeth more frequently than the men $(p<0.0001)$. The proportion of low frequency of toothbrushing was higher in men than women $(p<0.0001)$.

Table 2 shows the characteristics and Table 3 shows the preferences and lifestyle of the participants, stratified by the frequency of toothbrushing. Among men, the proportion of patients who consumed alcohol and lived alone was lower in patients who brushed their teeth twice or more a day than that in patients who brushed their teeth once a day. Among women, the total cholesterol level was higher in patients who brushed their teeth twice or more a day than that in patients who brushed their teeth once a day. The frequency of toothbrushing was similar in married persons and single persons among both men and women. No association was observed between the frequency of toothbrushing and lifestyle in women.

The logistic regression analysis (Table 4), after adjusting for the confounding factors, revealed that living alone (odds ratio 2.88 ; 95\% CI: $1.53-5.66$ ) was associated with the increased odds of the low frequency of toothbrushing (never/rarely or once a day) in men, but not in women.

\section{Discussion}

Recently, periodontal disease has drawn more attention than ever as it has been reported to be a risk factor for a wide variety of diseases, including CVD, dementia, and some types

Table 1. Characteristics of patients

\begin{tabular}{|c|c|c|c|c|}
\hline & All & Men & Women & $p$ \\
\hline$n$ & 624 & 376 & 248 & \\
\hline Age (years) & $67.6(10.9)$ & $67.3(11.2)$ & $68.1(10.4)$ & 0.35 \\
\hline Duration of diabetes (years) & $14.3(10.3)$ & $14.7(10.8)$ & $13.8(9.7)$ & 0.32 \\
\hline Body mass index $\left(\mathrm{kg} / \mathrm{m}^{2}\right)$ & $23.5(3.7)$ & $23.6(3.3)$ & $23.2(4.3)$ & 0.15 \\
\hline Glucose (mM) & $8.1(2.7)$ & $8.2(2.7)$ & $8.0(2.7)$ & 0.28 \\
\hline Hemoglobin A1c (\%) & $7.2(1.1)$ & $7.2(1.2)$ & $7.2(0.9)$ & 0.94 \\
\hline Total cholesterol (mM) & $4.8(0.8)$ & $4.7(0.8)$ & $5.0(0.8)$ & $<0.0001$ \\
\hline Triglycerides (mM) & $1.4(0.9)$ & $1.5(1.0)$ & $1.3(0.7)$ & 0.06 \\
\hline Uric acid $(\mu \mathrm{M})$ & $309.3(83.3)$ & $333.1(77.3)$ & $279.6(77.3)$ & $<0.0001$ \\
\hline Creatinine $(\mu \mathrm{M})$ & $79.6(79.6)$ & $88.4(88.4)$ & $70.7(61.9)$ & 0.0004 \\
\hline Smoking habit (past or current); $n(\%)$ & $372(59.6)$ & $312(83.0)$ & $60(24.2)$ & $<0.0001$ \\
\hline Hypertension; $n(\%)$ & $292(46.8)$ & $178(47.3)$ & $114(46.0)$ & 0.74 \\
\hline Hyperlipidemia; $n$ (\%) & $225(36.1)$ & $138(36.7)$ & $87(35.1)$ & 0.68 \\
\hline College education; $n(\%)$ & $220(35.3)$ & $175(46.5)$ & $45(18.1)$ & $<0.0001$ \\
\hline Employed; $n(\%)$ & $258(41.3)$ & $193(51.3)$ & $65(26.2)$ & $<0.0001$ \\
\hline Shift worker; $n(\%)$ & $31(5.0)$ & $22(5.9)$ & $9(3.6)$ & 0.21 \\
\hline Married; $n(\%)$ & $405(64.9)$ & $260(69.1)$ & $145(58.4)$ & 0.006 \\
\hline Living alone; $n(\%)$ & 87 (13.9) & $52(13.8)$ & $35(14.1)$ & 0.92 \\
\hline $\begin{array}{l}\text { Frequency of toothbrushing } \\
\text { (never or rarely/once a day/ } \geq \text { twice a day) }\end{array}$ & $43 / 194 / 387$ & 29/158/189 & $14 / 36 / 198$ & $<0.0001$ \\
\hline
\end{tabular}

Data are expressed as the mean (standard deviation) or absolute number. 
Table 2. Characteristics according to frequency of toothbrushing

\begin{tabular}{|c|c|c|c|c|c|c|c|c|}
\hline & \multicolumn{4}{|c|}{ Men } & \multicolumn{4}{|c|}{ Women } \\
\hline & Never/rarely & Once a day & $\geq$ Twice a day & $p$ & Never/rarely & Once a day & $\geq$ Twice a day & $p$ \\
\hline$n$ & 29 & 158 & 189 & & 14 & 36 & 198 & \\
\hline Age (years) & $68.8(12.9)$ & $67.3(10.6)$ & $68.8(12.9)$ & 0.74 & $70.3(8.3)$ & $69.8(10.6)$ & $67.7(10.5)$ & 0.37 \\
\hline Duration of diabetes (years) & $17.6(12.2)$ & $14.1(10.3)$ & $14.7(10.9)$ & 0.29 & $14.7(10.4)$ & $16.9(9.5)$ & $13.2(9.6)$ & 0.10 \\
\hline BMI $\left(\mathrm{kg} / \mathrm{m}^{2}\right)$ & $24.7(4.3)$ & $23.5(3.2)$ & $23.6(3.2)$ & 0.17 & $24.3(5.3)$ & $24.1(4.5)$ & $22.9(4.1)$ & 0.20 \\
\hline Glucose (mM) & $8.3(2.9)$ & $8.1(2.4)$ & $8.2(3.0)$ & 0.91 & $9.3(3.7)$ & $8.4(2.6)$ & $7.8(2.6)$ & 0.09 \\
\hline Hemoglobin A1c (\%) & $7.3(1.2)$ & $7.2(1.0)$ & $7.3(1.4)$ & 0.88 & $7.6(0.9)$ & $7.4(1.2)$ & $7.2(0.9)$ & 0.13 \\
\hline Total cholesterol (mM) & $4.4(0.6)$ & $4.7(0.8)$ & $4.7(0.9)$ & 0.06 & $5.2(0.6)$ & $4.6(0.8)^{*}$ & $5.0(0.8)$ & 0.01 \\
\hline Triglycerides (mM) & $1.4(0.6)$ & $1.5(1.3)$ & $1.4(0.9)$ & 0.62 & $1.6(0.8)$ & $1.4(0.6)$ & $1.3(0.6)$ & 0.32 \\
\hline Uric acid $(\mu \mathrm{M})$ & $345.0(77.3)$ & $327.1(71.4)$ & $333.1(77.3)$ & 0.49 & $267.7(65.4)$ & $309.3(83.3)$ & $279.6(77.3)$ & 0.08 \\
\hline Creatinine $(\mu \mathrm{M})$ & $79.6(17.7)$ & $97.2(114.9)$ & $88.4(53.0)$ & 0.74 & $61.9(17.7)$ & $70.7(26.5)$ & $70.7(61.9)$ & 0.84 \\
\hline Hypertension; $n(\%)$ & $15(51.7)$ & $71(44.9)$ & $92(48.7)$ & 0.70 & $5(35.7)$ & $20(55.6)$ & $89(45.0)$ & 0.37 \\
\hline Hyperlipidemia; $n$ (\%) & $9(36.7)$ & $66(41.8)$ & $63(33.3)$ & 0.22 & $6(42.9)$ & $13(36.1)$ & $68(34.3)$ & 0.80 \\
\hline
\end{tabular}

Data are expressed as the mean (standard deviation) or absolute number (percentage). BMI, body mass index. $P$ values are from ANOVA (for continuous data) or from chi-square tests (for categorical data). ${ }^{*} p<0.05$, vs $\geq$ twice a day.

Table 3. Preference and lifestyle according to frequency of toothbrushing

\begin{tabular}{|c|c|c|c|c|c|c|c|c|}
\hline & \multicolumn{4}{|c|}{ Men } & \multicolumn{4}{|c|}{ Women } \\
\hline Smoking habit past or current; $n(\%)$ & $24(82.8)$ & $135(85.4)$ & $153(80.9)$ & 0.54 & $4(28.6)$ & $13(36.1)$ & $43(21.7)$ & 0.17 \\
\hline Coffee drinking; $n(\%)$ & $16(55.2)$ & $108(68.4)$ & $134(70.9)$ & 0.24 & $10(71.4)$ & $20(55.6)$ & $122(61.6)$ & 0.57 \\
\hline Tea drinking; $n(\%)$ & $14(48.3)$ & $69(43.7)$ & $83(43.9)$ & 0.90 & $8(57.1)$ & $17(47.2)$ & $115(58.1)$ & 0.48 \\
\hline College education; $n(\%)$ & $11(37.9)$ & $71(44.9)$ & $93(49.2)$ & 0.46 & $2(14.3)$ & $6(16.7)$ & $37(18.7)$ & 0.89 \\
\hline Employed; $n(\%)$ & $14(48.3)$ & $80(50.6)$ & $99(52.4)$ & 0.89 & $3(21.4)$ & $7(19.4)$ & $55(27.8)$ & 0.53 \\
\hline Shift worker; $n(\%)$ & $0(0)$ & $10(6.3)$ & $12(6.4)$ & 0.38 & $0(0)$ & $0(0)$ & $9(4.6)$ & 0.31 \\
\hline Physical exercise; $n(\%)$ & $13(44.8)$ & $78(49.4)$ & $115(60.9)$ & 0.05 & $5(35.7)$ & $93(47.0)$ & $13(36.1)$ & 0.38 \\
\hline Married; $n(\%)$ & $150(51.7)$ & $109(69.0)$ & $136(72.0)$ & 0.09 & $9(64.3)$ & $21(58.3)$ & $115(58.1)$ & 0.90 \\
\hline Living alone; $n$ (\%) & $4(13.8)$ & $32(20.3) *$ & $16(8.5)$ & 0.007 & $3(21.4)$ & 5 (13.9) & $27(13.6)$ & 0.72 \\
\hline
\end{tabular}

Data are expressed as the absolute number (percentage). $P$ values are from chi-square tests $* p<0.05$, vs $\geq$ twice a day.

Table 4. Unadjusted odds ratios and multivariate adjusted odds ratios for low frequency of toothbrushing (never/rarely or once a day)

\begin{tabular}{lcccccc}
\hline \multicolumn{1}{c}{ Men } & Smoking (Ref. never) & $p$ & Alcohol consumption & $p$ & Living alone & $p$ \\
\hline Crude & $1.34(0.78-2.31)$ & 0.29 & $1.68(1.01-2.83)$ & 0.048 & $2.58(1.40-4.95)$ & 0.002 \\
Model 1 & $1.36(0.78-2.41)$ & 0.28 & $1.66(0.98-2.83)$ & 0.06 & $2.75(1.48-5.34)$ & 0.001 \\
Model 2 & $1.31(0.74-2.35)$ & 0.35 & $1.63(0.96-2.80)$ & 0.07 & $2.88(1.53-5.66)$ & 0.0009 \\
\hline \multicolumn{1}{c}{ Women } & Smoking (Ref. never) & $p$ & Alcohol consumption & $p$ & Living alone & $p$ \\
\hline Crude & $1.86(0.93-3.62)$ & 0.08 & $2.07(0.53-6.86)$ & 0.25 & $1.21(0.48-2.74)$ & 0.67 \\
Model 1 & $1.80(0.89-3.54)$ & 0.10 & $1.86(0.46-6.43)$ & 0.36 & $1.01(0.39-2.38)$ & 0.98 \\
Model 2 & $1.89(0.90-3.90)$ & 0.09 & $1.94(0.47-7.03)$ & 0.34 & $1.07(0.40-2.61)$ & 0.89 \\
\hline
\end{tabular}

Model 1 is adjusted for smoking habit, alcohol drinking, and living alone. Model 2 includes all variables in Model 1 plus age, body mass index, hemoglobin A1c, hypertension, and hyperlipidemia.

of cancers. ${ }^{(3,11,12)}$ In addition, several studies have revealed that diabetes is reciprocally associated with periodontal disease. .5, $, 13,14)$ The data suggest that better oral hygiene may contribute to better glycemic control, and vice versa. ${ }^{(6,13,15,16)}$ There are some studies showing the association between the frequency of toothbrushing and oral health. ${ }^{(17,18)}$ Moreover, it has been reported that the frequency of toothbrushing is associated with the risk of CVD and diabetes. ${ }^{(8,15,19)}$ Taken together, these reports suggest that good oral hygiene, which can be maintained by brushing the teeth on a daily basis, may be important for preventing co-morbid conditions, including CVD, in patients with diabetes. Concerning the frequency of toothbrushing, there 
are several characteristics worth pay attention to. According to the previous studies, those who brushed their teeth less often than twice a day were more likely to have diabetes and obesity, smokers and leading a sedentary life. ${ }^{(8)}$ In the present study, alcohol consumption was associated with the low frequency of toothbrushing on univariate analysis in men. However, smoking habit was not associated with the low frequency of toothbrushing on univariate analysis in men and women. With reference to a previous study, we have chosen smoking habit and alcohol consumption as co-factors over other lifestyle factors on multivariate analysis. ${ }^{(8)}$ Even more importantly, living alone significantly increased the odds of the low frequency of toothbrushing in men. To the best of our knowledge, this is the first study to report that living alone may be a characteristic associated with the low frequency of toothbrushing in men.

It has not yet been clear how living alone affected the practice of self-care. However, some studies have suggested that living alone affects both the physical and mental health. ${ }^{(20-23)}$ Holt-Lunstad et al. ${ }^{(22)}$ have reported that living alone is one of the risk factors for mortality. Hanna et al. ${ }^{(21)}$ have suggested that the persons who live alone have a lower diversity of food intake; lower consumption of fruits, vegetables, and fish; and a higher likelihood of having an unhealthy dietary pattern than those who do not live alone. In our study, living alone was associated with the low frequency of toothbrushing in men, but not in women. It has been generally considered that there are several differences in lifestyle between men and women. A previous study ${ }^{(21)}$ has reported that the men living alone were more often observed to have unhealthy diet than the women.

Of note, marital status had little association with the frequency of toothbrushing in our study. Therefore, the marital status may not be a matter of concern, but living alone is. There are some possible reasons why people live alone. Some might be forced to live apart from their family due to job transfer, whereas others might voluntarily choose to live alone. Even if someone is married, it does not always mean that he or she lives with their spouse or their family. Living with someone would encourage one to stick to the habits which help us stay healthy. Some people living alone might be less careful about their oral hygiene because there is no one at home who can warn them of their oral health. There were no studies which reported association between living alone and time for self-care. Previous studies ${ }^{(21,24)}$ have reported that people living alone tended to have poor healthy life. Therefore, we postulated that even if people living alone have more time for self-care, they may not spend spare time for selfcare. These tendencies may be particularly strong in men. In any case, living alone is associated with unhealthy lifestyle, including the frequency of toothbrushing.

Our study has a few limitations. First, there are many factors which can influence the motivation toward self-care; and every conceivable factor should ideally be identified and included in the analyses. We should evaluate the effect of other factors, such as economic status, the size of social network ties, and the frequency of going out or meeting someone else in our future studies. Second, a certain degree of recall bias might have crept in due to the use of self-administered questionnaire. However, this method is common, and the findings of our study are comparable to the results of other studies using the same method. One study using self-administered questionnaire reported a sex difference in the frequency of toothbrushing, which was consistent with our study. ${ }^{(8)}$ Third, the frequency of flossing is an important part of oral hygiene. Therefore, we may need to address this association as well. Unfortunately, however, we have no data regarding the frequency of flossing.

We suggested the importance of oral hygiene to prevent CVD. However, previous studies demonstrated an association only, and could not show a causative relationship between oral hygiene and the risk of CVD. There is currently no data demonstrating that intervening to improve oral health leads to reduced cardiovascular risk in not only people with diabetes but healthy people. It would be important to research whether oral health care can lead to prevent CVD. Further investigation using a larger population is crucial to prove and promote the importance of toothbrushing in those who have diabetes and healthy people.

In conclusion, we have deduced from the findings of our study that living alone is associated with the low frequency of toothbrushing in people with type 2 diabetes mellitus in men. It is important to focus on the frequency of toothbrushing and lifestyle in people with type 2 diabetes mellitus in clinical scene to reduce the risk of CVD. We might pay more attention to men living alone to provide a better oral health.

\section{Author Contributions}

YS researched data and wrote manuscript, $\mathrm{HO}$ and $\mathrm{MH}$ researched data and contributed to discussion, TO, YH, SM, TS, $\mathrm{NN}, \mathrm{EU}, \mathrm{MA}$, and MY researched data, MF researched data and reviewed/edited manuscript.

\section{Funding}

None.

\section{Abbreviations}

CI confidence interval

CVD cardiovascular disease

\section{Conflict of Interest}

$\mathrm{MH}$ has received grant support from Asahi Kasei Pharma, Mitsubishi Tanabe Pharma Corporation, Nippon Boehringer Ingelheim Co., Ltd., Sanofi K.K., Daiichi Sankyo Co., Ltd., Takeda Pharmaceutical Co., Ltd., Kyowa Kirin Co., Ltd., Sumitomo Dainippon Pharma Co., Ltd., Astellas Pharma Inc., Novo Nordisk Pharma Ltd., and Eli Lilly Japan K.K. YH has received grant support from Asahi Kasei Pharma and honoraria from Mitsubishi Tanabe Pharma Corporation and Novo Nordisk Pharma Ltd. SM has received honoraria from Novo Nordisk Pharma Ltd., Kowa Pharmaceutical Co., Ltd., AstraZeneca plc, Abbott Japan Co., Ltd., Ono Pharmaceutical Co., Ltd., Sumitomo Dainippon Pharma Co., Ltd. TS has received honoraria from Ono Pharmaceutical Co., Ltd., Mitsubishi Tanabe Pharma Co., Astellas Pharma Inc., Kyowa Hakko Kirin Co., Ltd., MSD K.K., Sanofi K.K., Kowa Pharmaceutical Co., Ltd., Novo Nordisk Pharma Ltd., Takeda Pharmaceutical Co., Ltd., Kissei Pharmaceutical Co., Ltd., Taisho Toyama Pharmaceutical Co., Ltd., Eli Lilly Japan K.K. NN has received honoraria from Novo Nordisk Pharma Ltd., Abbott Japan Co., Ltd., AstraZeneca plc, Kowa Pharmaceutical Co., Ltd., Ono Pharmaceutical Co., Ltd., Sumitomo Dainippon Pharma Co., Ltd. EU has received grant support from the Japanese Study Group for Physiology and Management of Blood Pressure, the Astellas Foundation for Research on Metabolic Disorders (Grant number: 4024). Donated Fund Laboratory of Diabetes therapeutics is an endowment department, supported with an unrestricted grant from Ono Pharmaceutical Co., Ltd., and received personal fees from AstraZeneca plc, Astellas Pharma Inc., Daiichi Sankyo Co., Ltd., Kowa Pharmaceutical Co., Ltd., Kyowa Hakko Kirin Company Ltd., MSD K.K., Mitsubishi Tanabe Pharma Corp., Takeda Pharmaceutical Co., Ltd., Taisho Toyama Pharmaceutical Co., Ltd., Novo Nordisk Pharma Ltd., Nippon Boehringer Ingelheim Co., Ltd., Johnson \& Johnson K.K., and Sumitomo Dainippon Pharma Co., Ltd. MA has received honoraria from Novo Nordisk Pharma Ltd., Abbott Japan Co., Ltd., AstraZeneca plc, Ono Pharmaceutical Co., Ltd., Kowa Pharmaceutical Co., Ltd., Sumitomo 
Dainippon Pharma Co., Ltd. MY has received personal fees from MSD K.K., Sumitomo Dainippon Pharma Co., Ltd., Kowa Co., Ltd., AstraZeneca plc, Takeda Pharmaceutical Co., Ltd, Kyowa Hakko Kirin Co., Ltd., from Daiichi Sankyo Co., Ltd., Kowa Pharmaceutical Co., Ltd., Ono Pharmaceutical Co., Ltd. MF has received grants from Nippon Boehringer Ingelheim Co., Ltd., Kissei Pharmaceutical Co., Ltd., Mitsubishi Tanabe Pharma Co., Daiichi Sankyo Co., Ltd., Takeda Pharmaceutical Co., Ltd., Sanofi K.K., Astellas Pharma Inc., MSD K.K., Kyowa Hakko Kirin Co., Ltd., Sumitomo Dainippon Pharma Co., Ltd., Novo Nordisk Pharma Ltd., Kowa Pharmaceutical Co., Ltd., Eli Lilly Japan K.K., Sanwa Kagaku Kenkyusho Co., Ltd., Taisho Pharmaceutical Co., Ltd., Ono Pharmaceutical Co., Ltd., Terumo Co.,

\section{References}

1 Isomaa B, Almgren P, Tuomi T, et al. Cardiovascular morbidity and mortality associated with the metabolic syndrome. Diabetes Care 2001; 24: 683-689.

2 Multiple Risk Factor Intervention Trial Research Group. Multiple risk factor intervention trial. Risk factor changes and mortality results. JAMA 1982; 248: 1465-1477.

3 Persson GR, Persson RE. Cardiovascular disease and periodontitis: an update on the associations and risk. J Clin Periodontol 2008; 35 (8 Suppl): 362-379.

4 Libby P, Ridker PM, Maseri A. Inflammation and atherosclerosis. Circulation 2002; 105: 1135-1143.

5 Wang TT, Chen TH, Wang PE, et al. A population-based study on the association between type 2 diabetes and periodontal disease in 12,123 middle-aged Taiwanese (KCIS No. 21). J Clin Periodontol 2009; 36: 372-379.

6 Demmer RT, Desvarieux M, Holtfreter B, et al. Periodontal status and A1C change: longitudinal results from the study of health in Pomerania (SHIP). Diabetes Care 2010; 33: 1037-1043.

7 Kobayashi Y, Niu K, Guan L, et al. Oral health behavior and metabolic syndrome and its components in adults. J Dent Res 2012; 91: 479-484.

8 de Oliveira C, Watt R, Hamer M. Toothbrushing, inflammation, and risk of cardiovascular disease: results from Scottish Health Survey. BMJ 2010; 340: c2451.

9 Ryu S, Chang Y, Kim DI, Kim WS, Suh BS. $\gamma$-Glutamyltransferase as a predictor of chronic kidney disease in nonhypertensive and nondiabetic Korean men. Clin Chem 2007; 53: 71-77.

10 Expert Committee on the Diagnosis and Classification of Diabetes Mellitus. Report of the expert committee on the diagnosis and classification of diabetes mellitus. Diabetes Care 2003; 26 Suppl 1: 5-20.

11 Tzeng NS, Chung CH, Yeh CB, et al. Are chronic periodontitis and gingivitis associated with dementia? A nationwide, retrospective, matched-cohort study in Taiwan. Neuroepidemiology 2016; 47: 82-93.

12 Michaud DS, Liu Y, Meyer M, Giovannucci E, Joshipura K. Periodontal disease, tooth loss, and cancer risk in male health professionals: a prospective cohort study. Lancet Oncol 2008; 9: 550-558.

13 Miyawaki A, Toyokawa S, Inoue K, Miyoshi Y, Kobayashi Y. Self-reported periodontitis and incident type 2 diabetes among male workers from a 5-year follow-up to MY Health Up Study. PLoS One 2016; 11: e0153464.

14 Nelson RG, Shlossman M, Budding LM, et al. Periodontal disease and NIDDM in Pima Indians. Diabetes Care 1990; 13: 836-840.

15 Kuwabara M, Motoki Y, Sato H, et al. Low frequency of toothbrushing prac-
Teijin Pharma Ltd., Nippon Chemiphar Co., Ltd., and Johnson \& Johnson K.K. Medical Company, and received honoraria from Nippon Boehringer Ingelheim Co., Ltd., Kissei Pharmaceutical Co., Ltd., Daiichi Sankyo Co., Ltd., Sanofi K.K., Mitsubishi Tanabe Pharma Corp., Takeda Pharmaceutical Co., Ltd., Astellas Pharma Inc., Kyowa Kirin Co., Ltd., MSD K.K., Sumitomo Dainippon Pharma Co., Ltd., Kowa Pharmaceutical Co., Ltd., Novo Nordisk Pharma Ltd., Ono Pharmaceutical Co., Ltd., Sanwa Kagaku Kenkyusho Co., Ltd., Eli Lilly Japan K.K., Taisho Pharmaceutical Co., Ltd., Bayer Yakuhin, Ltd., AstraZeneca K.K., Mochida Pharmaceutical Co., Ltd., and Combi Corp. The other authors have nothing to disclose.

tices is an independent risk factor for diabetes mellitus in male and dyslipidemia in female: a large-scale, 5-year cohort study in Japan. J Cardiol 2017; 70: $107-112$.

16 Sun WL, Chen LL, Zhang SZ, Wu YM, Ren YZ, Qin GM. Inflammatory cytokines, adiponectin, insulin resistance and metabolic control after periodontal intervention in patients with type 2 diabetes and chronic periodontitis. Intern Med 2011; 50: 1569-1574.

17 Kumar S, Tadakamadla J, Johnson NW. Effect of toothbrushing frequency on incidence and increment of dental caries: a systematic review and metaanalysis. J Dent Res 2016; 95: 1230-1236.

18 Gil GS, Morikava FS, Santin GC, Pintarelli TP, Fraiz FC, Ferreira FM. Reliability of self-reported toothbrushing frequency as an indicator for the assessment of oral hygiene in epidemiological research on caries in adolescents: a cross-sectional study. BMC Med Res Methodol 2015; 15: 14.

19 Chang Y, Woo HG, Park J, Lee JS, Song TJ. Improved oral hygiene care is associated with decreased risk of occurrence for atrial fibrillation and heart failure: a nationwide population-based cohort study. Eur J Prev Cardiol 2020; 27: 1835-1845.

20 Betihavas V, Frost SA, Newton PJ, et al. An absolute risk prediction model to determine unplanned cardiovascular readmissions for adults with chronic heart failure. Heart Lung Circ 2015; 24: 1068-1073.

21 Hanna KL, Collins PF. Relationship between living alone and food and nutrient intake. Nutr Rev 2015; 73: 594-611.

22 Holt-Lunstad J, Smith TB, Baker M, Harris T, Stephenson D. Loneliness and social isolation as risk factors for mortality: a meta-analytic review. Perspect Psychol Sci 2015; 10: 227-237.

23 Kim EJ, Jin BH. Comparison of oral health status and daily nutrient intake between elders who live alone and elders who live with family: based on the Korean National Health and Nutrition Examination Survey (KNHANES VI) (2013-2015). Gerodontology 2018; 35: 129-138.

24 Lidfeldt J, Nerbrand C, Samsioe G, Agardh CD. Women living alone have an increased risk to develop diabetes, which is explained mainly by lifestyle factors. Diabetes Care 2005; 28: 2531-2536.

This is an open access article distributed under the terms of the Creative Commons Attribution-NonCommercial-NoDerivatives License (http://creativecommons.org/licenses/by-nc-nd/4.0/). 